\title{
Proporción Salarial Hídrica Doméstica, propuesta de indicador entorno al gasto básico personal.
}

\author{
García Leyva, Alberto \\ Universidad Veracruzana, México \\ albergarcia@uv.mx
}

Resumen - La Proporción Salarial Hídrica Doméstica (PSHD) es un indicador propuesto en este artículo de investigación que mide el gasto personal en materia de consumo de agua para uso doméstico en proporción al salario mínimo mensual establecido en México, es un indicador diseñado para ser adaptado a otros espacios y a otras magnitudes de variables, de existir condiciones pertinentes, con el fin de crear información útil que agrega precios cercanos a la realidad y consecuentes para desarrollar una vida digna.

Palabras clave - Proporción Salarial Hídrica Doméstica (PSHD), Precio del Agua, Vida digna, Salario Mínimo, Indicadores.

Abstract - The Domestic Water Wage Rate (PSHD, by its initials in Spanish) is an indicator proposed in this research paper that measures personal spending on water consumption for domestic use in pro- portion to the monthly minimum wage established in Mexico, is an indicator designed to be adapted to others spaces and other magnitudes of variables, of existing relevant conditions, in order to create useful information that adds prices close to reality and consistent to develop a dignified life.

Keywords - Domestic Water Wage Rate; Water price; Dignified life; Minimum salary; Indicators.

\section{INTRODUCCIÓN}

El presente artículo de investigación está compuesto por una serie de secciones en las que primeramente, se expone el contexto en el cual se desarrolla el valor económico que se le otorga al bien natural del agua, posteriormente se propone una metodología para estimar el precio del consumo del agua per 
cápita dependiendo las tarifas y el consumo que una persona puede llegar a tener, este indicador lleva el nombre de Proporción Salarial Hídrica Doméstica (PSHD) y es construido con el fin de monitorear su comportamiento a través del tiempo, de las circustancias y factores que influyen en el valor económico del agua para uso doméstico.

El objetivo secundario que persigue esta investigación es el aportar nuevas metodologías y conceptos en torno al uso del agua, aportar un elemento más a los componentes que conforman el gasto de una persona, tomando a consideración central los precios de mercado actuales, que pueden ser comparados con el ingreso mínimo establecido que puede llegar a tener una persona habitante en la República Mexicana, de ahí la importante comparación e integración con el salario mínimo.

Para estimar el costo de vida digna de una persona es necesario acudir a una serie de cálculos relativos al consumo promedio diario durante un lapso de tiempo y lugar determinado, es preciso tomar en cuenta todos aquellos aspectos que significan un costo para una persona, se parte de la idea de que dicho costo debe ser en los precios en los que se encuentran los bienes y servicios en la realidad, en los que una persona los adquiere en la vida real en el diario vivir, aquellos precios que una persona puede adquirir tomando en cuenta los procesos inflacionarios en el tiempo actual.

Estimar el costo de vida digna de las personas ayuda a los tomadores de decisiones y público en general a poder comparar las condiciones en las que las personas viven en determinados lugares, en determinados lapsos temporales y comparar las condiciones reales con aquellas establecidas como idóneas para desarrollar una vida digna.

Ayuda además, para visualizar la problemática en la que se ve sometida la población en general y poder diseñar políticas públicas idóneas centradas en resolver los problemas que aquejan a la sociedad, que surgen a partir de la captura de las condiciones de vida reales en circunstancias existentes. 
Bajo ese orden de ideas, estimar el precio del agua relacionado con un consumo per cápita del mismo y corresponderlo con el salario mínimo que una persona en México puede tener, va enfocado en mostrar que porcentaje va destinado en obtener un servicio fundamental para el desarrollo de la vida cotidiana y doméstica, que impulsa la obtención de desarrollar una vida aceptable.

\section{CONTEXTO DE LA VALORACIÓN Y EL PRECIO DEL AGUA}

El cálculo del porcentaje del salario relacionado al precio del consumo de agua para uso doméstico es de relevancia, ya que permite visualizar la magnitud de adquirir un servicio indispensable, en el que las personas y las familias deben incurrir para el desarrollo de sus actividades diarias, es un precio del que no se puede eludir, en el que además, se ven inmiscuidos tópicos de suma importancia como el cuidado al medio ambiente y el uso adecuado de los recursos naturales, sobre todo cuando el recurso del agua es de suma relevancia para la vida en general, a este escenario se le agrega la situación de que en algunos lugares ya empieza a escasear este importante recurso.

El agua tiene una serie de características que lo vuelven un símbolo de suma importancia, pertenece a la cultura social más que cualquier otro recurso natural, diferentes hechos económicos políticos y sociales que se encuentran afines con la gestión del agua, obedecen a diferentes circunstancias geográficas, demográficas e hídricas en diferentes ámbitos y regiones (Rolland, Louise; Vega Cárdenas, 2010).

Faltan una serie de elementos para determinar el valor real del agua, ya que ésta pertenece a un ciclo natural que no obedece a un sistema económico con objetivos comerciales, no se ha podido encontrar aquellos elementos que vuelven sostenible la explotación de este recurso natural (Torres-Sombra, Jesús; García-Salazar, José A.; García-Mata, Roberto; Matus-Gardea \& González-Estrada, Elizabeth; Pérez-Zamorano, 2013). 
Dependiendo de la capacidad de pago que tienen ciertos sectores de la sociedad para obtener una serie de bienes y servicios, como lo es el agua, es el precio que se le atribuye a dichos insumos, esto bajo el concepto del principio de equidad (Del Villar, 2010).

Para el caso mexicano se puede encontrar qué estas herramientas de mercado que utilizan precios y tarifas no se han utilizado de manera continua por lo que los gobiernos optan por estrategias enfocadas en un aumento de la oferta que trae consigo grandes inversiones que tienen perjuicios para el medio ambiente, que al no tener el resultado esperado utilizan la herramienta del racionamiento y tandeo del agua (Sainz, Jaime; Becerra, 2003).

En torno a esta serie de factores es como se determina el precio del agua, aunque existe una cadena de características que rodean el proceso de distribución del agua en determinadas ciudades de la República Mexicana que conlleva a una alteración importante en los precios de este importante recurso natural.
Solís M., 2005 menciona que los precios del agua en los países de la OCDE han aumentado de manera importante en los últimos 10 años, esto incluye también al saneamiento, de la información que se encuentra disponible para algunos países de la OCDE se observaron un aumento en el precio del agua y 6 países mostraron un aumento del $6 \%$ o más al año con precios no fijos.

Castro Rosales, Gregorio; Sisto, 2015 mencionaron en una investigación del manejo del agua urbana en México, que un aumento en el precio del agua no tendría consecuencias servibles como herramienta para el control de la demanda del bien líquido y no creará oportunidades para tener alternativas para desarrollar nuevas fuentes de agua, la demanda del agua es significativamente perceptible al número de consumidores que a su precio.

Bajo este contexto, el valor económico que se le otorga al agua en las ciudades mexicanas para uso doméstico se ve sometido a una serie de alteraciones que deben ser monitoreadas constante- 
mente con el afán de ser comparadas con diferentes circunstancias económicas y sociales que pueden determinar su valor, con el objetivo de identificar cuáles son aquellas que tienen mayor influencia sobre el bien y poder realizar así, una serie de estudios que pueden evidenciar ciertas causalidades o generar insumos para ejercicios predictivos entre otras muchas acciones.

\section{PRECIO DEL CONSUMO DEL}

\section{AGUA}

Ante las aseveraciones antes expuestas, surge la necesidad de calcular una serie de indicadores en torno al precio y consumo del agua que sea capaz de actualizarse por lo menos cada mes, para poder así visualizar la evolución y el comportamiento del impacto económico que tiene este importante recurso natural para evidenciar las políticas que se construyen entorno al agua, un indicador de esta naturaleza sirve además para monitorear el impacto en la economía de las personas.

Partiendo de estos conceptos, se desea estimar el gasto que una persona tiene en su consumo de agua de uso doméstico como porcentaje del salario mínimo en México, con el fin de obtener una proporción de lo que las personas destinan en un servicio indispensable para el diario vivir, y que forma parte de una serie de gastos en los que la población mexicana debe incurrir para tener una vida digna. Se muestra a continuación una propuesta de indicador que captura estas características, con detalle de las variables que lo componen;

\section{PROPORCIÓN SALARIAL}

\section{HíDRICA DOMÉSTICA (PSHD)}

Porcentaje del precio del consumo del agua doméstico per cápita con respecto al salario mínimo.

\section{La Proporción Salarial Hídrica} Doméstica está compuesta por las siguientes variables:

$$
P S H D=((P M 3 \alpha C A P) / S M) 100
$$

\section{Nomenclatura:}

PSHD = Proporción Salarial Hídrica Doméstica

$S M=$ Salario mínimo mensual

CAP = Consumo de agua per cápita PM3 = Precio de la tarifa de servicio de agua del metro cúbico 
La Proporción Salarial Hídrica Doméstica (PSHD) se calcula de la siguiente manera; se obtiene el valor per cápita de consumo de agua doméstico y se estima el valor a pagar según el precio de la tarifa de agua, de tal manera que la variable del costo de los metros cúbicos del agua está en proporción al consumo per cápita estimado, posteriormente es divido entre el salario mínimo mensual, el resultado de esta operación es multiplicado por cien y se obtiene así la Proporción Salarial Hídrica Doméstica, el resultado se expresa en porcentaje.

\section{SALARIO MÍNIMO}

Para obtener la proporción de gasto en agua domestico de una persona durante un mes, se procede a calcular el salario mínimo mensual, que en esta investigación y para el indicador que se propone se calcula con la reproducción del salario mínimo diario por 30 días, el salario mínimo establecido será el publicado por la secretaria de hacienda y crédito público SHCP y el servicio de administración tributaria SAT que son establecidos por la Comisión Nacional de los Salarios Mínimos mediante las publicaciones en el Diario Oficial de la Federación.

\section{CONSUMO DE AGUA POR}

\section{PERSONA}

El consumo de agua por persona es un factor determinante en la conformación del indicador aquí propuesto, es indispensable tener una cantidad con la cual se calculará el indicador que este respaldado por fuentes confiables, de instituciones que se encargan del monitoreo de información relativa al agua.

El cálculo del consumo de agua por persona se da a partir de los resultados concretos que se esperan obtener, ya que de otro modo pude existir información sesgada ocasionada por supuestos distantes de la realidad, bajo este orden de ideas se toma solo el consumo per cápita de agua para uso doméstico ya que puede ser comparado con otras variables pertinentes, cuyo nivel de medida sea el per cápita y el consumo que las personas tienen sea en sus hogares, omitiendo así el consumo indirecto del agua, decisión de importancia si se desea comparar, estimar y/o calcular con variables que pueden ser adaptadas a la misma unidad de medida. 
En el libro titulado Huella hídrica en México en el contexto de Norteamérica de AgroDer y WWF México del año 2012 se menciona en la página número 37 que el consumo per cápita que México tiene es de 1978 metros cúbicos por año con información de FAO, en ese mismo documento (página 30) de AgroDer SC con información de FAOSTAT, WFN y SIAP se menciona que el $5 \%$ del consumo es doméstico, que es el dispendio pertinente para la conformación del indicador, por lo que el consumo per cápita mensual esta entre 8.1285 y 8.2416 metros cúbicos mensuales de agua según se calcule;

\section{Opción 1}

$1978(0.05)=98.9$ de consumo domestico

$98.9 / 365$ días $=0.27095$

$0.27095^{*} 30$ días $=8.1285$

\section{Opción 2}

$1978(0.05)=98.9$ de consumo domestico $98.9 / 12$ meses $=8.2416$

Para esta investigación se tomara el valor intermedio de entre estas dos cantidades expuestas dando como resultado 8.1850 metros cúbicos al mes per cápita. El cálculo se determina mensualmente para fines comparativos y estimativos con las variables que se expondrán más adelante.

\section{PRECIO DEL AGUA}

El precio del agua será el establecido para la ciudad de Xalapa, determinado como supuesto, pero la conformación de la formula anteriormente expuesta, está diseñado para que se pueda adaptar a toda aquella ciudad de México siempre y cuando se tenga la información pertinente, entre los que se encuentran lo relativo al precio del agua, se debe contar con las tarifas del precio de la misma de manera mensual.

Para el caso ejemplo de la ciudad de Xalapa, ubicada en el estado de Veracruz en el Golfo de México, las tarifas de agua que se expondrán a continuación (ver tabla 1) es la que se publicaron mensualmente en la página de internet de la Comisión Municipal de Agua Potable y Saneamiento de Xalapa, Veracruz, en el apartado referente a las tarifas del agua, mismo en donde se pueden descargar o visualizar los tabulados referidos a los precios de diversos índoles que son relativos a servicios de agua y saneamiento, se publica una serie de tabulados por mes, en el apartado de la página web, se encuentran 
desde enero del 2014 a diciembre de 2017 hasta la fecha de realización de este artículo de investigación, cada uno de los apartados de estos tabulados tienen el formato similar, variando ligeramente en algunas ediciones con respecto a años anteriores, el mes que no se encuentra disponible es el de di- ciembre del año 2016, con estas publicaciones se espera que publiquen información para los años posteriores, para poder calcular y estimar la Proporción Salarial Hídrica Doméstica y darle así un seguimiento a esta indicador.

\section{Tabla 1. Tarifa del agua del mes de diciembre de 2017 de la CMAS Xalapa}

\begin{tabular}{|c|c|c|c|c|}
\hline $\begin{array}{l}\text { Rango } \\
\text { (metro } \\
\text { cúbico) }\end{array}$ & Popular & $\begin{array}{l}\text { Interés } \\
\text { Social }\end{array}$ & $\begin{array}{c}\text { Doméstico } \\
\text { Medio }\end{array}$ & Residencial \\
\hline $1(0-10)$ & $\$ 56.39$ & $\$ 80.59$ & $\$ 102.17$ & $\$ 120.81$ \\
\hline $2(11-20)$ & $\$ 5.66$ & $\$ 8.15$ & $\$ 10.90$ & $\$ 13.13$ \\
\hline $3(21-30)$ & $\$ 6.36$ & $\$ 10.28$ & $\$ 13.54$ & $\$ 16.44$ \\
\hline $4(31-40)$ & $\$ 6.53$ & $\$ 10.83$ & $\$ 13.96$ & $\$ 16.92$ \\
\hline $5(41-60)$ & $\$ 6.96$ & $\$ 11.26$ & $\$ 14.67$ & $\$ 17.89$ \\
\hline $6(61-80)$ & $\$ 7.38$ & $\$ 13.11$ & $\$ 15.66$ & $\$ 19.02$ \\
\hline $7(81-100)$ & $\$ 7.82$ & $\$ 14.05$ & $\$ 17.53$ & $\$ 21.43$ \\
\hline $8(101-120)$ & $\$ 8.83$ & $\$ 14.41$ & $\$ 18.03$ & $\$ 22.07$ \\
\hline $9(121-150)$ & $\$ 9.51$ & $\$ 15.70$ & $\$ 19.49$ & $\$ 23.79$ \\
\hline $10(151-200)$ & $\$ 10.77$ & $\$ 17.53$ & $\$ 21.91$ & $\$ 26.71$ \\
\hline $11(201-250)$ & $\$ 12.02$ & $\$ 19.72$ & $\$ 24.37$ & $\$ 29.65$ \\
\hline $12(251-\ldots)$ & $\$ 12.25$ & $\$ 20.01$ & $\$ 24.79$ & $\$ 30.34$ \\
\hline
\end{tabular}

Fuente: Elaboración propia con tabla de la Comisión Municipal de Agua Potable y Saneamiento de Xalapa, Veracruz (CMAS). Xalapa H. Avuntamiento. Recuperado el 22 de Enero del 2018 de; https://cmasxalapa.gob.mx/tarifas/ 
En la tabla de tarifas se muestran cuatro rubros los cuales son pertinentes para la investigación y el cálculo de la Proporción salarial hídrica doméstica, los otros apartados del cuadro que muestra la CMAS de Xalapa son los referentes a comercial A, comercial B, institución pública e industrial, pero por la orientación del indicador no son tomados en cuenta, lo que deja al interés solo de los rubros mostrados en el cuadro 1, relativos a Interés Social, Domestico Medio, y Residencial, en los cuales se pueden tomar para el calcula del indicador propuesto, puede ser dependiendo la zona y sector de la población que se desee explorar, calcular un promedio por la ciudad, o calcular para aquella zona que predomine.

En la tabla 1 se observa una marcada diferencia entre la zona de interés social y residencial, ya que en el primer apartado relativo al rango número 1 que va de 0 a 10 metros cúbicos el precio en popular es de 56 pesos con 39 centavos y para residencial es de 120 pesos con 81 centavos, una diferencia de más del doble, misma divergencia en los precios de los consiguientes rangos en donde también supera más del doble.
Considerando las marcadas diferencias entre zonas para la tarifa del agua, se puede realizar una ponderación entre las 4 zonas pertinentes aquí mostradas, bajo la opción de sumar las tarifas por rango y dividirlas entre el número de zonas disponibles, en este caso cuatro, de tal manera de obtener un promedio por cada rango.

Otra opción es tomar las tarifas de la zona popular y determinar la Proporción Salarial Hídrica Doméstica mínima de la ciudad y posteriormente calcular de nuevo el indicador con los valores de la zona residencial y estimar así la cantidad máxima de Proporción salarial hídrica doméstica, con el objeto de establecer un rango en el que la población de la ciudad se verá ubicada dependiendo las variables.

El indicador de la Proporción Salarial Hídrica Doméstica está diseñado para modificar con las cantidades de las variables que lo componen, con el fin de estimar la cantidad a analizar sin que se vea sometido a cantidades fijas de manera rigurosa, que pueden ser no representativas para las investigaciones a realizar. Se trata de una 
propuesta de metodología de indicador que puede ser adaptada según la investigación.

\section{CONCLUSIÓN}

Los factores que influyen en el precio del agua son de diversas índoles que pasan por circunstancias sociales, económicas y políticas diversas. Ante estos cambios provocados por dichos factores, los precios de un bien tan necesario como el agua, influyen necesariamente en el gasto que tienen las personas y familias de una ciudad determinada.

De ahí la necesidad de monitorear la evolución de estos cambios, para poder identificar cuáles son aquellas variables qué aportan una significativa causalidad y correlación con el precio de este importante recurso natural.

El indicador aquí propuesto denominado Proporción Salarial Hídrica Doméstica, es un indicador que surge de la idea de representar mediante un número el porcentaje destinado a obtener dicho bien, que resulta ser determinante para desa- rrollar las actividades diarias de una persona promedio.

El indicador propuesto está diseñado con una facultad adaptativa para cualquier ciudad, tarifa de agua, magnitud de consumo y tipo de salario al que se desea comparar, siempre y cuando existan datos disponibles y que sean confiables. Se puede realizar una batería de datos utilizando la misma metodología de obtención del resultado para que tenga fines comparativos, ya sea con otras ciudades, otros tiempos, salarios, tarifas y consumos.

La Proporción Salarial Hídrica Doméstica surge como un esfuerzo para estimar el valor monetario que significa tener una vida digna con la obtención de bienes y servicios que permitan desarrollarla, además que se calculen con precios que se encuentran disponibles en el mercado en el tiempo actual y con valores actuales, este indicador está planeado para ser agregado a otra serie de indicadores para medir el costo de vida digna de las personas en México, tomando fragmentos importantes en los que intervienen para desarrollar una vida adecuada, 
que van desde los precios de los alimentos hasta la obtención se servicios básicos, siempre comparado con el salario mínimo establecido en el país.

\section{BIBLIOGRAFÍA}

AgroDer, 2012. Huella Hídrica en México en el contexto de Norteamérica. WWF México y AgroDer. México DF. Recuperado el 18 de Enero del 2018 de; http://www.huellahidrica.org/Reports/AgroDer,\%202012.\%20HueIla $\% 20 \mathrm{~h} \% \mathrm{C} 3 \%$ ADdrica $\% 20$ en $\% 20 \mathrm{M} \% \mathrm{C}$ $3 \%$ A9xico.pdf

Comisión Municipal de Agua Potable y Saneamiento de Xalapa, Veracruz (CMAS). Xalapa H. Ayuntamiento. Recuperado el 22 de Enero del 2018 de; https://cmasxalapa.gob.mx/tarifas/

SAT. Servicio de Administración Tributaria. SHCP. Secretaría de Hacienda y Crédito Público.

Castro Rosales, Gregorio; Sisto, N. P. (2015). Precio y manejo del agua urbana en México. Nóesis. Revista de Ciencias Sociales Y Humanidades, 24(47), 222-242. Retrieved from http://www.redalyc .org/articulo. $.0 a ?$ id $=85932588010$

Del Villar, A. (2010). Los precios de los servicios del agua. Un análisis prospectivo de demanda sobre los usos domésticos. Estudios de Economía Aplicada, 28(2), 333-355. Retrieved from http://www.redalyc.org/pdf/301/30120357005.pdf

Rolland, Louise; Vega Cárdenas, Y. (2010). La gestión del agua en México. Polis: Investigación Y Análisis Sociopolítico Y Psicosocial, 6(2), 155-188. Retrieved from http://www.redalyc.org/pdf/726/72618890006.pdf

Sainz, Jaime; Becerra, M. (2003). Los conflictos por el agua en México. Gaceta Ecológica, (67), 61-68. Retrieved from http://www.redalyc.org/articulo. oa? $\mathrm{id}=53906705$

Solís M., L. (2005). La escasez, el costo y el precio del agua en México. Economíaunam, 2(6), 24-42. Retrieved from http://www.redalyc.org/articulo.oa?id=363542887002

Torres-Sombra, Jesús; García-Salazar, José A.; García-Mata, Roberto; MatusGardea, J., \& González-Estrada, Elizabeth; Pérez-Zamorano, A. (2013). RESPUESTA DE LA DEMANDA DE AGUAA CAMBIOS EN EL PRECIO: UN ESTUDIO POR TIPO DE CONSUMIDOR EN EL NORTE DE SINALOA, MÉXICO. Agrociencia, 47(3), 293-307. Retrieved from http://www.redalyc.org/articulo.oa?id=30226978008 